\title{
Performance Evaluation of Downlink Design Based on Free Space Optical System
}

\author{
A. A. Anis ${ }^{1, *}$, C.B.M.Rashidi ${ }^{1}$, A. K. Rahman ${ }^{2}$, S.A.Aljunid ${ }^{1}$ and Yaakob $S^{3}$ \\ ${ }^{1}$ Advance Communication Engineering, Centre of Excellence School of Computer \& Communication Eng, Universit Malaysia Perlis, \\ (UniMAP) Kangar, 01000, Kangar, Perlis \\ ${ }^{2}$ Deparment of Electrical and Electronic Eng, Universiti Malaysia Sarawak, (UNIMAS), Sarawak \\ ${ }^{3}$ Deparment of Computer and Communication Systems Eng, Faculty of Eng, Universiti Putra Malaysia (UPM), Serdang, Selangor
}

\begin{abstract}
In this paper, the performance of downlink design utilizing the free space optic (FSO) is presented. The permissible BER of $10^{-58}$ is achieved when the system utilized $40 \mathrm{~cm}$ aperture diameter. The highest system sensitivity will lead to less error and enable more transmission output. The result revealed, at a bit rate $155 \mathrm{Mbps}$ and effective received power $-27.5 \mathrm{dBm}$, FSO downlink system at bit rate $155 \mathrm{Mbps}$ improved by $13 \%$ as compared to $1.5 \mathrm{Gbps}$ and $2.5 \mathrm{Gbps}$ in single transmission line. It is truly shown that, the FSO system utilized $155 \mathrm{Mbp} / \mathrm{s}$ with $40 \mathrm{~cm}$ aperture diameter shown good performance of a reliable FSO downlink system.
\end{abstract}

\section{Introduction}

Free space optical (FSO) has to be included in the set of answers for qualifying the bandwidth requirements of the modern internet. FSO system is an incredible alternative to overcome the bottleneck broadband connectivity problem. Recently, FSO is very active with numerous fundamental and technological problems to enhance its performance in a wide situations range. The swift continuity in the increasing of data rate transmission by FSO communication system in both long- and short-range applications implementing the rapid development of the system performance. Figure 1 shows a basic scheme of the FSO communication system with optical wireless and diverse user connectivity.

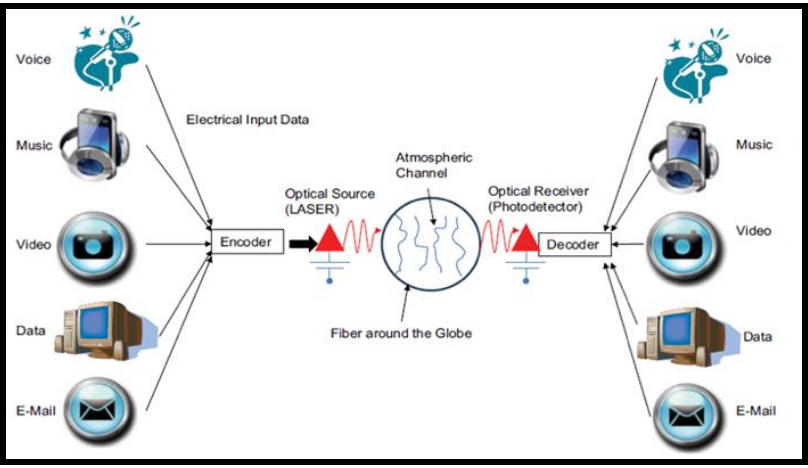

Fig. 1. A basic scheme of the FSO communication system with optical wireless and diverse user connectivity [1]

Various FSO link conditions must fulfil multiple system needs. These involve various propagation channel characteristics, different technique of transmission, and detection methods. The advantage of indoor applications of FSO communications such as secure communications and sensor networking over short ranges is drawing attention globally. It is made as a non-interfering replacement to RF communications [1]. Speeds usually up to $2.5 \mathrm{Gbps}$ are achievable and ranges up to a few $\mathrm{km}$, depends on technology and conditions of the atmosphere [2]. In a FSO link the transmitters transmit high power light. The power light however appears as low power light at the receiver after transferring through the air.. The link margin of the connection shows the light amount received by an optical terminal over the lowest value crucial to maintain the link active. Fig. 2 displays the basic communication system which is consisting of a transmitter, a receiver, and a communication channel.

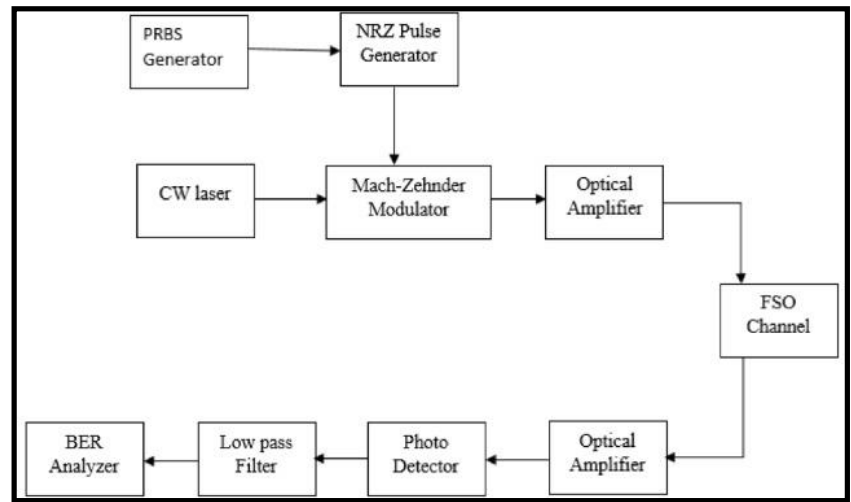

Fig. 2. The basic communication system [3]

There are some parameters related to the performance degradation of a FSO link. They are the

\footnotetext{
* Corresponding author: anis afina@ymail.com
} 
link range between optical transceiver, line of sight, alignment of optical transceiver, atmospheric conditions, water vapour and hot air, strong electromagnetic interference, and optical signal wavelength [4]. An expendable microwave link is inevitably crucial, as the laser link can stop working under various changing conditions. Numerous investigations and applications of FSO have been reported [5-6]. A laser beam faces power loss due to various factors as it propagates through the air. One of them is absorption and scattering loss as defined in Beer's Law [7]. Absorption happens due to the photons of the beam bumping with haze particles. This phenomenon can reduce the link margin, distance and the availability of the link [8]. Scattering is the dispersion of a radiation beam due to physical interactions.

\section{Bit Error Rate Simulation Analysis}

The structural design of FSO can be categorized into three types of connections. They Point-to-Point connections, Point-to-Multipoint connections, and Multipoint-to-Multipoint connections. Point-to-Point connections consist of one transmitter and one receiver. This type of connection provides greater bandwidth although their availability is lower. The second connection is Point-to-Multipoint connections. They consist of one transmitter and multiple receivers. This second design provides low cost connections. Its advantage is that it facilitates node addition of the network. Even so, this type of connection provides poor bandwidth level compared to the former. The third design of FSO connection is Multipoint- to- Multipoint connections. This connections utilize multiple receivers and transmitters. They are also called as Mesh Network which provides redundancy and better reliability with easy node addition. Nonetheless, these connections have a significant disadvantage which shorter is distance limitation than other FSO designs. The FSO networks are shown in Figure 3, Figure 4 and Figure 5.

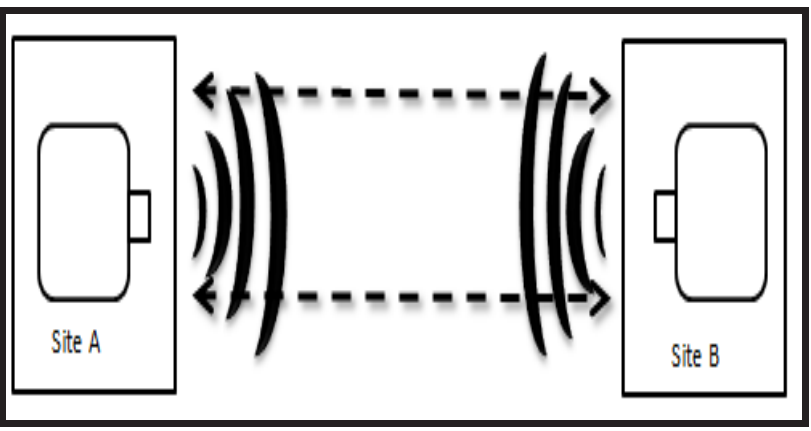

Fig. 3. Point-to-Point Connection

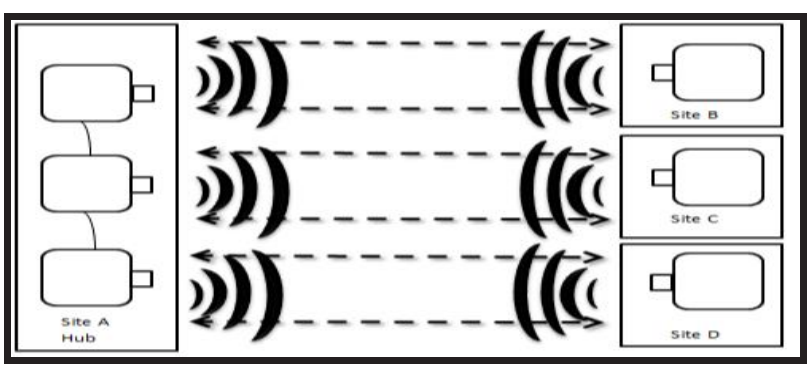

Fig. 4. Point-to-Multipoint Connection

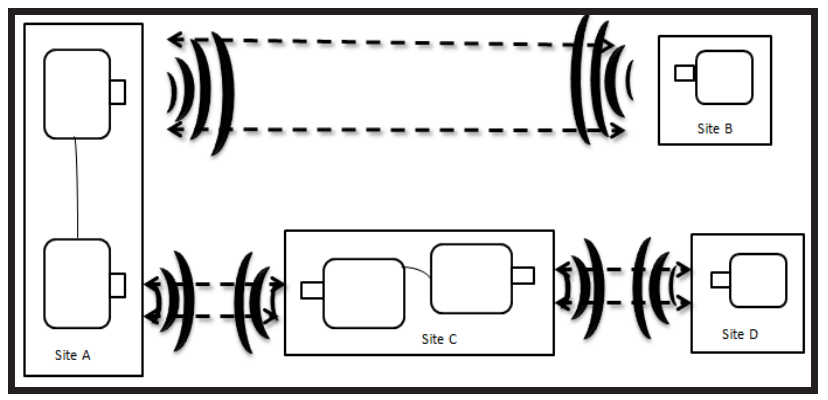

Fig. 5. Multipoint-to-Multipoint Connection

FSO systems provide numerous advantages to the users. Examples are being low start-up and economical, swift deployment, and high bandwidths due to the technology optical nature[9]. The optical bandwidth can reach up to $1 \mathrm{Gbps}$ or more. It is primarily to assist wide bandwidth range over a long distance communication [10]. Although at high bandwidth, long distance cost efficient communication the environmental factors such as weather linked factors continue as major challenges to minimize and may limit the data rate and link availability via LOS (Line of Sight) [11]. Recent studies suggest ways to solve LOS line of sight problems with minimal bit-error-rate (BER) performance in FSO communication system under worst interference conditions [12]. But the increasing of laser power can resolve this problem up to some point [13]. As the transmitter reaching maximum power limit, the bandwidth or transmission distance of the physical links which are under severe weather conditions such as heavy haze has to be reduced. This will cause some alterations in the structures of FSO networks. Thus, the effectiveness and performance of Free Space Optical network will be affected.

\section{Bit Error Rate Simulation Analysis}

Bit error rate (BER) is one of the main elements of FSO communication system. BER can be estimated using equation 1

$B E R \approx \frac{N e}{N b}$

where,

$\mathrm{Ne}=$ received error bits number

$\mathrm{Nb}=$ transmitted bits for a period 
Generally, the BER values for a digital transmission system are usually specified and influenced on the Signal-to-Noise-Ratio (SNR) of the optical receiver.

This feature determined the probability of errors when one bit of data is being transmitted. The internal noise of the optical receiver affects the value of BER [14]. The value of BER can be calculated using the following formula.

$B E R=\frac{1}{2} \operatorname{erfcQ}$

However in this paper, the results for tht BER are obtained from simulation using OptiSys ${ }^{\mathrm{TM}}$ simulation tool. The data collected are analysed in the form of graph and evaluated in the section below.

\section{Results and Discussion}

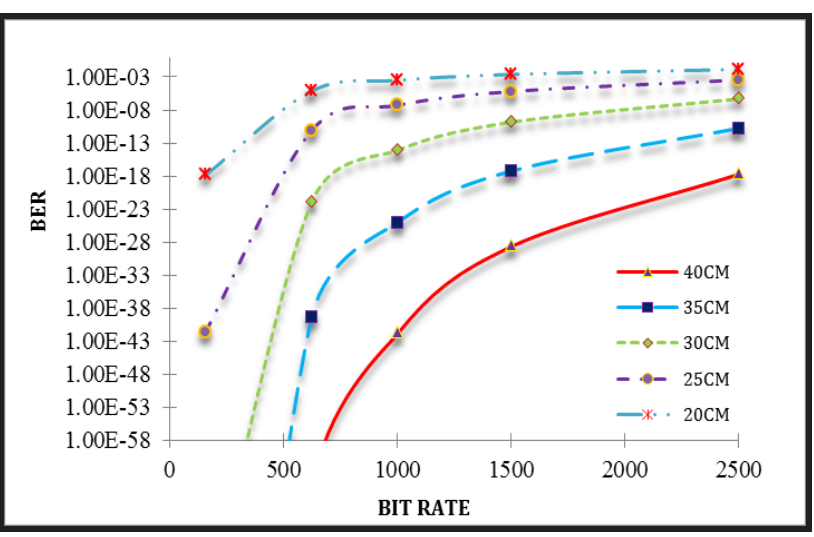

Fig. 6. Graph of bit rate against bit error rate (BER) with different receiver aperture diameter

Figure 6 demonstrate the relationship between bit error rate (BER) and bit rate using apertures diameter which ranges from 20 to $40 \mathrm{~cm}$. From the graph, it can be seen that the bit error rate increases when there is bit rate increment. It is observed that the receiver aperture diameter of $40 \mathrm{~cm}$ has achieved the lowest bit error rate throughout the investigation (from bit rate $155 \mathrm{mbps}$ to $2.5 \mathrm{gbps}$ ) compared to aperture diameter of $20,25,30$, and $35 \mathrm{~cm}$ respectively. The FSO with receiver aperture diameter $40 \mathrm{~cm}$ has the lowest BER of $10^{-58}$ at $155 \mathrm{mbps}$. Then it finishes at BER $10^{-18}$ with bit rate of 2.5 Gbps. For receiver aperture diameter of $20 \mathrm{~cm}$, the BER is $10^{-18}$ at the bit rate $155 \mathrm{mbps}$ until BER $10^{-2}$ at bit rate of 2.5 Gbps. From the figure, it can be concluded that by increasing the receiver aperture diameter, the receiver's sensitivity is also increased. Higher sensitivity will allow more outputs and reduce the error received by the system. This will directly improve the system at high performance.

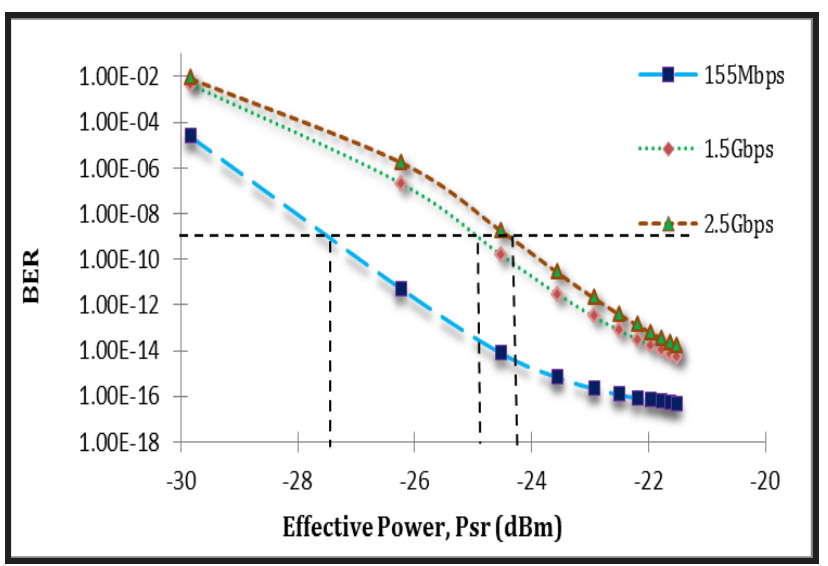

Fig. 7. Graph of bit error rate (BER) versus effective power with different bit rate

Figure 7 shows plot a BER versus effective power for bit rate $155 \mathrm{Mbps}, 1.5 \mathrm{Gbps}$ and $2.5 \mathrm{Gbps}$, respectively by considering all at range $1 \mathrm{~km}$ and receiver aperture diameter at $20 \mathrm{~cm}$. the value caused of effective power $P_{s r}$ varies from $-30 \mathrm{dBm}$ to $-20 \mathrm{dBm}$. It can be seen that, the bit rate $155 \mathrm{Mbps}$ have a better performance contrast with the bit rate $1.5 \mathrm{Gbps}$ and $2.5 \mathrm{Gbp}$, respectively, when the effective receives power $P_{s r}$ for bit rate 1.5 Gbps and $2.5 \mathrm{Gbps}$ are larger (i.e. when $P_{s r}>-25 \mathrm{dBm}$ ). The effective receives power for bit rate $155 \mathrm{Mbps}$ is $27.5 \mathrm{dBm}$ at $\mathrm{BER}=10^{-9}$ better than bit rate $1.5 \mathrm{Gbps}$ and $2.5 \mathrm{Gbps}$ are $-24.8 \mathrm{dBm}$ and $-24.4 \mathrm{dBm}$ respectively. The effective receiver power for bit rate $155 \mathrm{Mbps}$ is the lower receiver power that can be reached to the receiver as compared with the bit rate $1.5 \mathrm{Gbps}$ and $2.5 \mathrm{Gbps}$. The bit rate 155 Mbps proved that high bit rate can enhance the transmission link by $13 \%$ compared to bit rate $1.5 \mathrm{Gbps}$ and $2.5 \mathrm{Gbps}$ are $2 \%$ impeovement.

\section{Conclusions}

The downlink FSO communication system with 155 Mbps bit rate at BER $10^{-9}$ is best suited in comparison with a bit rate $1.5 \mathrm{Gbps}$ and $2.5 \mathrm{Gbps}$, respectively. The result had shown the bit rate $155 \mathrm{Mbps}$ with low BER $10^{-9}$ can obtain optical receive power $P_{s r}-27.5 \mathrm{dBm}$. In addition, by using large aperture diameter, consequently, will improve system sensitivity.

\section{References}

1. Majumdar, A.K.: Fundamentals of Free-Space Optical (FSO) Communication System.(2015)

2. Pacheco de Carvalho, J.A.R., Marques, N., Veiga, H., Ribeiro Pacheco, C.F., Reis, A.D.: Extended Field Performance Evaluation of a Gbps FSO Link. ICETE. (2012) 439-446

3. Chauhan, N.R., Vala, M.K.: System Design and Performance Analysis on the Free Space Optics (FSO) System in Atmospheric Turbulence. IRJET, Vol. 4. (2017) 1789-1793 
4. Rockwell, D.A., Mecherle, G.S.: Wavelength Selection for Optical Wireless Communication Systems. SPIE, Vol. 4530. (2001) 27-35

5. D’Amico, M., Leva, A., Micheli, B.: Free Space Optics Communication Systems. First Results From a Pilot Field Trial in the Surrounding Area of Milan, Italy. IEEE. (2003) 305-307

6. Löschnigg, M., Mandl, P., Leitgeb, E.: Long-term Performance Observation of a Free Space Optics Link. 10th International Conference on Telecommunications. (2009) 305-310

7. Kaushal, H., Kaddoum, G.: Free Space Optical Communication: Challenges and Mitigation Techniques. (2015)

8. Alkholidi, A., Altowij, A.: Effect of Clear Atmospheric Turbulence on Quality of Free Space Optical Communications in Western Asia. (2012) 41-74

9. Srinivasan, R., Vijayaraj, A.: Mobile Communication Implementation Techniques to Improve Last Mile High Speed FSO Communication. (2011) 55-63

10. Kedar, D., Arnon, S.: Urban optical wireless communication networks: the main challenges and possible solutions. (2004)

11. Liu, Q., Qiao, C., Mitchell, G., Stanton, S.: Optical wireless communication networks for first- and lastmile broadband access. Journal of Optical Networking. (2005) 807-828

12. Arnon, S., Kopeika, N.S.: Adaptive optical transmitter and receiver for space communication through thin clouds. Applied Optics. (1997) 1991987

13. CBM Rashidi, SA Aljunid, F Ghani, HA Fadhil, MS Anuar, AR Arief, Optik-International Journal for Light and Electron Optics 125 (17), 4889-4894

14. Vorontsov, M.A., Dudorov, V.V., Zyryanova, M.O.,Kolosov, V.V., Filimonov, G.A.: Bit error rate in free-space optical communication systems with a partially coherent transmitting beam. Atmospheric and Oceanic Optics, Vol. 26. (2013) 185-189. 